\title{
Warrants and Deception in Computer Mediated Communication
}

\author{
Darcy Warkentin ${ }^{1}$, Michael Woodworth ${ }^{1}$, Jeffrey T. Hancock ${ }^{2}$, \& Nicole Cormier $^{3}$ \\ ${ }^{1}$ Department of Psychology \\ UBC Okanagan \\ ${ }^{2}$ Department of Communication \\ ${ }^{3}$ Department of Psychology \\ Cornell University \\ Kelowna, BC, Canada \\ dlwn64@hotmail.com \\ Ithaca, NY, USA \\ jeff.hancock@cornell.edu \\ Ryerson University
Toronto, ON, Canada
ncormier@psych.ryerson.ca
}

michael.woodworth@ubc.ca

\begin{abstract}
This article explores the operation of warrants, connections between online and real-world identities, on deceptive behavior in computer-mediated communication. A survey of 132 participants assessed three types of warrants (the use of a real name, a photo, and the presence of real-world acquaintances) in five different media: IM, Forums, Chat, Social Networking Sites (SNS) and Email. The effect of warrants on lies about demographic information (e.g., age, gender, education, etc.), one's interests (e.g., religion, music preferences, etc.), and the seriousness of lies was assessed. Overall, deception was observed most frequently in Chat and least often in SNS and Email. The relationship between warrants and deception was negative and linear, with warrants suppressing the frequency and seriousness of deception regardless of medium, although real-world acquaintances were especially powerful in constraining deception in SNS and emails.
\end{abstract}

\section{Author Keywords}

Deception, anonymity, CMC, warrants, computermediated communication

\section{ACM Classification Keywords}

J.4 Social and behavioral sciences: psychology

\section{General Terms \\ Experimentation, Human Factors, Theory}

\section{INTRODUCTION}

Anonymity has been one of the most intriguing social psychological aspects of computer-mediated communication (CMC). The effects of anonymity have not been simple. In some cases, anonymity has been

Permission to make digital or hard copies of all or part of this work $f$ personal or classroom use is granted without fee provided that copies a not made or distributed for profit or commercial advantage and that copi bear this notice and the full citation on the first page. To copy otherwis or republish, to post on servers or to redistribute to lists, requires pri specific permission and/or a fee.

CSCW 2010, February 6-10, 2010, Savannah, Georgia, USA.

Copyright 2010 ACM 978-1-60558-795-0/10/02...\$10.00. linked to anti-normative behavior, including deceptive behavior, yet at other times, to more honest behavior [5].

In the present study, we examine a concept related to anonymity, warrants, which are connections between our real world identity and our online identity [3]. Following other recent work, we hypothesize that warrants reduce and constrain deception [1]. Specifically, the use of real names, photographs, and the presence of real-world acquaintances, which all connect us to our real-world identities, should reduce the frequency of lies and constrain how serious they are. Because previous research suggests that we lie differently in different media [2], we compare the operation of these warrants across five of the most common forms of CMC: Instant Messaging (IM), Internet Forums, Chat Rooms, Social Networking Sites (SNS) and Email.

\section{Features, warrants and deception}

Previous research has found that deception patterns change across media, including frequency and content. For instance, in diary studies email conversations involve fewer lies than other media, like instant messaging [2]. One line of research has argued that deception is affected by specific features of the medium that are particularly important for deception: synchronicity (real-time interactivity), persistence (extent to which a record is generated), and distribution (physical separation). Nonpersistent and synchronous media, such as the telephone, have been linked to higher rates of deception, while persistent and asynchronous media, such as email, have lower rates.

This approach, however, does not consider the relative anonymity of different media, which intuitively should be important for deception. As noted earlier, the concept of a warrant has recently been proposed to function as a constraint on deception [1]. Previous work on warrants, however, has focused on how warrants can affect our perception of information about others. For instance, we tend to trust other-generated remarks over self-generated remarks for comments on Facebook [4]. Here we argue that warrants can also affect our behavior, in particular, our deceptive practices. Given that warrants are pieces of information that provide a link to a user's real world 
identity, we argue that warrants should reduce deception because they increase the likelihood of being caught out in a lie. By examining the effect of warrants on deceptive behavior, we hope to extend the warranting framework beyond perceptions to practices.

We examine the effect of three warrants on types and seriousness of deceptive behavior across media: name, photo and acquaintance warrants.

Name warrants refer to the use of one's real name in a communication space. When one's name is furnished then any online behavior can be potentially linked to one's real-world identity. If this is the case, then name warrants should constrain deception.

Photo warrants refer to recognizable pictures that can link visual identity to real-world identity. Over the last several years posting profile pictures in various communication media has become common. The most obvious are SNS profiles, in which each profile has photographs of the user. However, most other media can also include photographs, including Chat, Forums, Instant Messaging and web-based email (e.g., gmail.com). The presence of a photo should act as a warrant and reduce deception in media in which the photo is present.

Acquaintance warrants refer to the degree to which a user's communication partners in a given medium are known to the user. Some media, such as Chat and Forums, tend to involve interactions between individuals who do not know each other outside that space. Other media, such as SNS, are primarily about communicating with friends, family and other acquaintances that are known to the user in the real world.

Given that acquaintance warrants tie a user's online identity and behaviors directly to their real world identity, acquaintance warrants should be particularly powerful at suppressing deception. One example of warrants reducing deception comes from a study of online dating profiles, in which daters who told friends about their online dating activities posted more accurate photos than daters who hid their online dating from friends [4]

\section{Present study}

If these warrants affect deception as we predict, then the presence of warrants should reduce deception, regardless of what medium they are in. Importantly, and in contrast to a feature-based approach, warrants are not necessarily a feature of a medium (one can upload or not upload a photo even if that feature is available). To the best of our knowledge, no research has examined the presence of warrants across media, or their impact on deception behavior.

The present study surveyed participants on the presence of warrants in each of five media: IM, Forums, Chat, SNS and Email. Participants also reported on their deceptive behavior in each of these media, including deceptions about demographic information (e.g., age, gender, etc.) and their interests (e.g., religion, hobbies, etc), allowing us to compare deception across media. Our primary hypothesis is that warrants should reduce deception and help explain any differences in deception across media.

\section{METHODS}

\section{Participants}

Participants $(n=132)$ completed an anonymous webbased survey in exchange for $0.5 \%$ bonus credit, applicable to participating undergraduate psychology courses. Due to a data collection error, demographic data were obtained from only 84 participants: 61 female and 23 male, with an average age of 21.2 years.

\section{Procedure}

Each participant filled out a web-based survey at their convenience. The questionnaire was divided into sections according to type of communication media: email, IM, forums, chat rooms, and social networking sites. The same set of questions was presented for each different medium to allow comparisons between the five.

First, participants completed items about deception in several different topical categories: "How often have you lied, misrepresented or omitted information so as to present less than the truth to someone you were communicating with on the following topic (1-5 scale):" age, gender, occupation, living arrangements, education, religion, hobbies, musical preferences, and other.

We transformed these items to a dichotomous response (lied on this item or did not). The dichotomous responses were subjected to an exploratory factor analysis with varimax rotation. The analysis revealed two factors with items loading over .6: a demographic deception factor (age, gender, occupation living arrangement and education) and an interests deception factor (religion, hobbies, musical preferences and other).

Finally, we also asked participants to rate the seriousness of their lies in each medium ("In general how serious do you think the lies you tell on this medium are?" 1-7 scale).

In the next set of questions, participants completed questions related to the three types of warrants (name, photo, acquaintances) in each medium. For each medium these questions assessed the presence of a name warrant (used their real name or not), a photo warrant (used a real photo of themselves or not), and an acquaintance warrant (whether most of the people they regularly talked to they knew in real life). 


\begin{tabular}{|lccccc|}
\hline & $\mathrm{IM}$ & Forum & Chat & SNS & Email \\
\hline Warrant & $\mathrm{n}=129$ & $\mathrm{n}=101$ & $\mathrm{n}=78$ & $\mathrm{n}=126$ & $\mathrm{n}=131$ \\
\hline Name & $74.4 \%$ & $0 \%$ & $18.0 \%$ & $97.6 \%$ & $92.3 \%$ \\
Photo & $58.1 \%$ & $13.0 \%$ & $10.3 \%$ & $92.9 \%$ & $8.5 \%$ \\
Acquaintance & $89.2 \%$ & $23.0 \%$ & $24.4 \%$ & $96.0 \%$ & $91.5 \%$ \\
\hline Mean Total Warrants & $2.22(.07)$ & $1.36(.06)$ & $0.53(.09)$ & $2.87(.04)$ & $1.92(.05)$ \\
\hline Deception Type & & & & & \\
\hline Demographic & $1.11(.10)$ & $0.97(.13)$ & $1.62(.15)$ & $0.42(.07)$ & $0.47(.08)$ \\
Interests & $1.72(.12)$ & $1.45(.13)$ & $1.86(.16)$ & $0.98(.11)$ & $0.65(.10)$ \\
Seriousness & $1.94(.14)$ & $1.97(.15)$ & $2.51(.19)$ & $1.86(.14)$ & $2.00(.15)$ \\
\hline
\end{tabular}

TABLE 1. PRESENCE OF WARRANTS AND CHARACTERISTICS OF DECEPTION ACROSS MEDIA

\section{RESULTS AND DISCUSSION}

\section{Analytic approach}

The data were analyzed with a mixed model approach, with medium type nested within participant. This analytic approach allowed for us to account for the nonindependence of data for participants across media. The descriptive data across media are described in Table 1

\section{Deception across media}

Consistent with previous research [2], the medium affected the nature of deception. The medium significantly affected the frequency of demographic deceptions, $(4,252.4)=19.99, p<.001$, with post hoc $(p<$ .01 ) analyses revealing the following order of demographic deception across media: chat $>\mathrm{IM}=$ Forum $>$ SNS $=$ Email. The medium also significantly affected deceptions about interests, $F(4,259.98)=18.20, \mathrm{p}<.001$, in this order: $\mathrm{IM}=$ Forum $=$ Chat $>$ SNS $=$ Email. Finally, the medium only marginally affected the seriousness of deceptions, $F(4,184.1)=2.13 p=.08$. This effect was driven by Chat, which involved more serious lies than any other medium. Taken together, these data suggest that SNS and Email involved the fewest lies overall, while Chat involved the most and the most serious.

\section{Warrants across media}

Our next set of analyses examined the presence of warrants across media (see Table 1). Because the presence of warrants was dichotomous (present vs. not present), non-parametric tests were used to compare across media (Friedman's test).

The percentage of participants who used their real name varied significantly across media, $\chi^{2}(4)=152.97, p<$ .001 . Name warrants were most frequently present in SNS and Email, followed by IM, and the least in Forum and Chat. The percentage of participants who included a photo in their communication also varied significantly across media, $\chi^{2}(4)=131.88, p<.001$. Photo warrants were most often present in SNS, followed by IM, and least in Forum, Chat and Email. Lastly, the number of people known varied by medium, $\chi^{2}(4)=146.67, p<$ .001 . Acquaintance warrants were most often present in SNS, Email and IM, and least present in Forum and Chat.

While the warrants are clearly qualitatively different, we sought to see if they had an additive effect. To calculate the most warranted medium we summed the presence of the name, photo and acquaintance warrants by participant, with a possible range of 0 (no warrants) to 3 (all warrants). The mean number of warrants differed significantly across medium, $F(4,25.98)=216.12 p<.001$. Post hoc analyses $(p<.001)$ revealed that each medium differed from the other in the following order: SNS $>$ IM $>$ Email $>$ Forum $>$ Chat, suggesting that SNS is the most warranted environment while Chat is the least.

\section{Warrants and deception}

The previous analyses reveal that 1) the most warrants are present in SNS, followed by IM, Email, Forum and least of all chat, and 2) the fewest deceptions were observed in Email and SNS, followed by IM and Forum, and the most in Chat. These rankings suggest that more warrants correspond with fewer and less serious deception.

To test this pattern, we conducted several analyses. The first set of analyses tested the relationship between total warrants and our three measures of deception, regardless of medium. As expected, the more warrants present the fewer demographic lies, $F(3,302.71)=14.96, p<.001$ and the fewer deceptions about interests, $F(3,347.55)=5.59$, $p<.001$. The negative relationship between warrants and deceptions was linear in both cases. Finally, the number of warrants present also constrained the seriousness of lies, $F(3,341.63)=2.89, p=.04$, again in a linear fashion.

Did the specific type of warrant (e.g., name, photo, acquaintance) differentially impact deception behavior? We expected that the acquaintance warrant, which most 
directly links online behavior with real-world identity, to be the most powerful constraint on deception. This was the case. When each of the warrants was entered simultaneously into the model, only the acquaintance warrant significantly affected deception. Fewer lies where told when more communication partners were known in real life (demographic: $M=0.77 ; S E=.08$; interests: $M=$ $1.22, S E=.09)$ than when few or none were known (demographic: $M=1.23 ; S E=.11$; interests: $M=1.64, S E$ $=.12)$, demographic: $F(1,416.57)=13.99, p<.001$; interests: $F(1,380.17)=9.66, p<.002$. Furthermore, the acquaintance warrant marginally constrained the seriousness of lies, $F(1,378.43)=3.06, p=.08$. These effects did not hold for either of the other warrants, suggesting that the acquaintance warrant accounts for the most variance in deception behavior.

\section{Warrants, medium and deception}

Our last question was concerned with whether the specific kind of warrant (e.g., name, photo, acquaintance) interacted with the medium on deceptive behavior. That is, do warrants operate differently in different media? The results revealed no interactions between warrant and medium on any of the deception characteristics save one: the acquaintance warrant interacted with medium on the seriousness of the lies, $F(5,2.86)=2.86, p=.02$. For SNS and Email only, the acquaintance warrant significantly constrained the seriousness of lies. This effect did not obtain for the other media. Taken together, these data suggest that the acquaintance warrant was more important in affecting the seriousness of SNS and email lies than other warrants, but only for these media.

\section{CONCLUSION}

Our primary objective was to examine how warrants affect deceptive behavior in CMC. As we expected, warrants were present differentially across media, with user's having the most warrants in SNS and the least in Chat. In fact, SNS are highly warranted - over ninety percent of participants had all three types of warrants present in SNS.

Our expectation that warrants would suppress deception was supported by the data. In general, the more warrants participants presented the fewer lies reported, regardless of medium. Acquaintance warrants were particularly powerful at constraining deception. When we pit the warrants against each other in predicting deception behavior, the acquaintance warrant was consistently a significant factor while the others were not. This finding is consistent with the idea that people seek to avoid having deceptions caught by friends and family, and is consistent with work examining the impact of network ties on deception [1].
The pattern of deception across the five media was also of interest. Replicating previous feature-based approach research [2], persistent media (e.g., email) involved less deception than ephemeral media (IM, Chat). However, warranting appeared to provide a better account of deception across media than a strictly features based approach. For instance, because Internet Forums leave a record and are asynchronous, a feature based approach would predict relatively low rates of deception. This was not the case - Forums were ranked second in deceptiveness, consistent with a warranting analysis.

There may be some utility, however, in considering both features of the medium and the warrants presented by users. For instance, although Email did not contain as many warrants due to the absence of photos, it ranked as low in deception, perhaps in part because of its high persistence and low synchronicity. Future experimental research is required to tease apart the influences of features and warrants more directly.

The present study provides important insights into the nature of online deception. Clarifying and understanding the link between media type, warrants and online deception may serve to improve the safety and well-being of various users of online media. There is also much we can learn about deception from the social networking model. Designs that encourage the use of real names, photos, and links to social networks, can serve to constrain both the frequency and seriousness of deception in online spaces.

\section{REFERENCES}

1. Guillory, J. \& Hancock, J.T. (2009). Online Social Networks as a Constraint to Deception in Online Resumes. Paper presented at the 59th International Communication Association Conference, Chicago, IL.

2. Hancock, J. T., Thom-Santelli, J., \& Ritchie, T. (2004). Deception and design: The impact of communication technology on lying behaviour. Proc. CHI conference 2004, 130-126. Cornell University

3. Walther, J. B., \& Parks, M. R. (2002). Cues filtered out, cues filtered in: Computer-mediated communication and relationships. In M. L. Knapp \& J. A. Daly (Eds.), Handbook of interpersonal communication (3rd ed., pp. 529-563). Thousand Oaks, CA: Sage.

4. Walther, J.B., Van Der Heide, B., Hamel, L.M., \& Schulman, H.C. (2009). Self-Generated Versus OtherGenerated Statements and Impressions in ComputerMediated Communication: A Test of Warranting Theory Using Facebook. Comm Res, 36, 229-253

5. Whitty, M.T., \& Joinson, A.N. (2008). Truth, Trust and Lies on the Internet. Psychology Press. 